\title{
An iterative procedure for solving the Riccati equation
}

\author{
$A_{2} R-R A_{1}=A_{3}+R A_{4} R$ \\ by \\ M. Thamban NAir (Madras)
}

\begin{abstract}
Let $X_{1}$ and $X_{2}$ be complex Banach spaces, and let $A_{1} \in \mathrm{BL}\left(X_{1}\right), A_{2} \in$ $\mathrm{BL}\left(X_{2}\right), A_{3} \in \mathrm{BL}\left(X_{1}, X_{2}\right)$ and $A_{4} \in \mathrm{BL}\left(X_{2}, X_{1}\right)$. We propose an iterative procedure which is a modified form of Newton's iterations for obtaining approximations for the solution $R \in \mathrm{BL}\left(X_{1}, X_{2}\right)$ of the Riccati equation $A_{2} R-R A_{1}=A_{3}+R A_{4} R$, and show that the convergence of the method is quadratic. The advantage of the present procedure is that the conditions imposed on the operators $A_{1}, A_{2}, A_{3}, A_{4}$ are weaker than the corresponding conditions for Newton's iterations, considered earlier by Demmel (1987), Nair (1989) and Nair (1990) in the context of obtaining error bounds for approximate spectral elements. Also, we discuss an application of the procedure to spectral approximation under perturbations of the operator.
\end{abstract}

1. Introduction. Let $X_{1}$ and $X_{2}$ be complex Banach spaces, and let

$$
A_{1}: X_{1} \rightarrow X_{1}, \quad A_{2}: X_{2} \rightarrow X_{2}, \quad A_{3}: X_{1} \rightarrow X_{2}, \quad A_{4}: X_{2} \rightarrow X_{1}
$$

be bounded linear operators. We shall specify conditions on the above operators so that the Riccati equation

$$
A_{2} R-R A_{1}=A_{3}+R A_{4} R
$$

has a unique solution $R \in \mathrm{BL}\left(X_{1}, X_{2}\right)$, and also introduce an iterative procedure for obtaining $R_{k} \in \mathrm{BL}\left(X_{1}, X_{2}\right), k=1,2, \ldots$, such that

$$
\left\|R-\left(R_{1}+\ldots+R_{k}\right)\right\| \rightarrow 0 \quad \text { as } k \rightarrow \infty,
$$

and

$$
\left\|R-\left(R_{1}+\ldots+R_{k+1}\right)\right\| \leq \alpha\left\|R-\left(R_{1}+\ldots+R_{k}\right)\right\|^{2}
$$

for some $\alpha>0$.

The above Riccati equation arises naturally when looking for an invariant subspace of a bounded linear operator having a specified complementary subspace. To see this let $A: X \rightarrow X$ be a bounded linear operator on a complex Banach space $X$ and $P_{0}: X \rightarrow X$ be a (bounded linear) projection

2000 Mathematics Subject Classification: 45B05, 47A10, 65F35, 65R20. 
operator. Suppose

$$
X_{1}=P_{0}(X), \quad X_{2}=\left(I-P_{0}\right)(X) .
$$

Let $R: X_{1} \rightarrow X_{2}$ be a bounded linear operator and $P: X \rightarrow X$ be defined by

$$
P x=P_{0} x+R P_{0} x, \quad x \in X .
$$

Then it can be easily seen that $P$ is a projection operator along $X_{2}$. Moreover, $P(X)$ is invariant under $A$ if and only if $R$ satisfies the Riccati equation

$$
A_{2} R-R A_{1}=A_{3}+R A_{4} R,
$$

where $A_{1}: X_{1} \rightarrow X_{1}, A_{2}: X_{2} \rightarrow X_{2}, A_{3}: X_{1} \rightarrow X_{2}, A_{4}: X_{2} \rightarrow X_{1}$ are given by

$A_{1}=\left.P_{0} A\right|_{X_{1}}, \quad A_{2}=\left.\left(I-P_{0}\right) A\right|_{X_{2}}, \quad A_{3}=\left.\left(I-P_{0}\right) A\right|_{X_{1}}, \quad A_{4}=\left.P_{0} A\right|_{X_{2}}$. (For details one may see [5] or [6].) Thus the problem of finding the invariant subspace $P(X)$ is equivalent to solving the Riccati equation (1.1).

Now suppose that $\left(S_{k}\right)$ is a sequence of bounded linear operators from $X_{1}$ to $X_{2}$ such that

$$
\left\|R-S_{k}\right\| \rightarrow 0 \quad \text { as } k \rightarrow \infty
$$

where $R: X_{1} \rightarrow X_{2}$ satisfies (1.1). Then the operators

$$
P_{k}:=P_{0}+S_{k} P_{0}, \quad k=1,2, \ldots,
$$

are projections along $X_{2}$, and we have

$$
\left\|P-P_{k}\right\| \rightarrow 0 \quad \text { as } k \rightarrow \infty .
$$

Recall from Kato [2] that, for subspaces $M_{1}$ and $M_{2}$ of $X$, if we define the distance from $M_{1}$ to $M_{2}$ as

$$
\operatorname{sep}\left(M_{1}, M_{2}\right):=\sup \left\{\operatorname{dist}\left(x, M_{2}\right): x \in M_{1},\|x\|=1\right\},
$$

then $\operatorname{gap}\left(M_{1}, M_{2}\right)$, the gap between $M_{1}$ and $M_{2}$, is given by

$$
\operatorname{gap}\left(M_{1}, M_{2}\right)=\max \left\{\operatorname{sep}\left(M_{1}, M_{2}\right), \operatorname{sep}\left(M_{2}, M_{1}\right)\right\} .
$$

If we set

$$
M_{k}=P_{k}(X), \quad M=P(X),
$$

then it follows that

$$
\operatorname{sep}\left(M_{k}, M\right) \leq\left\|R-S_{k}\right\|, \quad \operatorname{gap}\left(M_{k}, M\right) \leq\left\|P-P_{k}\right\|=\left\|\left(R-S_{k}\right) P_{0}\right\| .
$$

Thus the convergence of $\left(S_{k}\right)$ to $R$ implies the convergence of $\left(M_{k}\right)$ to $M$ in the sense of sep and gap.

In practical situations, what one would initially have is an approximate spectral subspace $M_{0}$, in the sense that $M_{0}$ is a spectral subspace of a perturbed operator $A_{0}$. In such a situation, the projection $P_{0}$ may be the spectral projection with $M_{0}=P_{0}(X)$ associated with a spectral set $\Lambda_{0}$ of $A_{0}$. 
Then, what one would like to look for is a spectral subspace $M$ associated with a spectral set $\Lambda$ of $A$.

The application of our results, with suitable conditions on $M_{0}$, does in fact give a sequence $\left(M_{k}\right)$ of subspaces and a sequence $\left(\Lambda_{k}\right)$ of closed subsets of the complex plane defined by

$$
\Lambda_{k}=\sigma\left(\left.P_{0} A\right|_{M_{k}}\right), \quad k=1,2, \ldots,
$$

such that

$$
\begin{gathered}
\operatorname{sep}\left(M_{k}, M\right) \rightarrow 0, \quad \operatorname{gap}\left(M_{k}, M\right) \rightarrow 0, \\
\operatorname{sep}\left(\Lambda_{k}, \Lambda\right):=\sup \left\{\operatorname{dist}(\lambda, \Lambda): \lambda \in \Lambda_{k}\right\} \rightarrow 0
\end{gathered}
$$

as $k \rightarrow \infty$, where $M$ is the spectral subspace of $A$ associated with a spectral set $\Lambda$.

In all the above discussed results, the crucial role is played by the size of the quantity

$$
\varepsilon:=\frac{\left\|A_{3}\right\| \cdot\left\|A_{4}\right\|}{\delta^{2}} .
$$

Here, $\delta:=\operatorname{sep}\left(A_{1}, A_{2}\right)$ is defined by

$$
\operatorname{sep}\left(A_{1}, A_{2}\right)= \begin{cases}0 & \text { if } 0 \in \sigma(T) \\ 1 /\left\|T^{-1}\right\| & \text { otherwise }\end{cases}
$$

where $T: \mathrm{BL}\left(X_{1}, X_{2}\right) \rightarrow \mathrm{BL}\left(X_{1}, X_{2}\right)$ is defined by

$$
T(B)=A_{2} B-B A_{1}, \quad B \in \operatorname{BL}\left(X_{1}, X_{2}\right) .
$$

It is to be remarked that iterative procedures for approximately solving Riccati equation (1.1) and the corresponding problem of approximating an invariant subspace/spectral subspace have been considered by many authors (see e.g. Stewart [9], Demmel [1], Nair [5], [6], [7]). The results in Stewart [9] and Nair [5] are based on a Piccard-type iteration, namely,

$$
A_{2} R_{k+1}-R_{k+1} A_{1}=A_{3}+R_{k} A_{4} R_{k}, \quad R_{0}=0,
$$

and provide linear convergence under the assumption that $\varepsilon<1 / 4$. Demmel [1] and Nair [7] use Newton's method, namely,

$$
\left(A_{2}-R_{k} A_{4}\right) R_{k+1}-R_{k+1}\left(A_{1}+A_{4} R_{k}\right)=A_{3}-R_{k} A_{4} R_{k}, \quad R_{0}=0,
$$

and obtain quadratic convergence whenever $\varepsilon<1 / 12$. Note that the quadratic convergence of the iteration in (1.3) is achieved not only by imposing a stronger assumption on the matrices $A_{1}, A_{2}, A_{3}, A_{4}$, but also by requiring to solve the equation each time with new coefficient matrices.

In [6], the author considered two iterative procedures which modify the iterations (1.2) and (1.3) and obtained improved estimates together with linear convergence if $\varepsilon<1 / 4$ and quadratic convergence in the case of $\varepsilon<(\sqrt{3}-1) / 4$. 
The new iterative procedure suggested in this paper is

$$
A_{2}^{(k)} R_{k+1}-R_{k+1} A_{1}^{(k)}=R_{k} A_{4} R_{k},
$$

where

$$
A_{1}^{(k)}=A_{1}^{(k-1)}-A_{4} R_{k}, \quad A_{2}^{(k)}=A_{2}^{(k-1)}-R_{k} A_{4},
$$

and $R_{1}$ is the unique solution of

$$
A_{2}^{(0)} R_{1}-R_{1} A_{1}^{(0)}=A_{3} \quad \text { with } \quad A_{1}^{(0)}=A_{1}, A_{2}^{(0)}=A_{2} .
$$

Of course, in this procedure, the coefficient matrices also have to be computed at each step of the iteration. But the advantage over existing Newtontype methods is that the conditions on the matrices $A_{1}, A_{2}, A_{3}, A_{4}$ are weaker. We show, in fact, that the iterative procedure (1.4) is valid for $\varepsilon<1 / 4$, and it provides quadratic convergence whenever $\varepsilon<3 / 16$. Observe that

$$
\frac{1}{12}<\frac{\sqrt{3}-1}{4}<\frac{3}{16}<\frac{1}{4}
$$

2. Basic definitions and preliminary results. Let $X, X_{1}$ and $X_{2}$ be complex Banach spaces. We denote by $\operatorname{BL}\left(X_{1}, X_{2}\right)$ the space of all bounded linear operators from $X_{1}$ into $X_{2}$, and denote $\operatorname{BL}(X, X)$ by $\operatorname{BL}(X)$.

The following result is, by now, well known in the literature. The "if" part was proved by Rosenblum [8] for $A_{1}, A_{2}$ in a Banach algebra, and the "only if" part was proved by Stewart [9] while obtaining error bounds for invariant subspaces of a closed linear operator in a Hilbert space. Stewart's result was extended by Nair [4] (quoted in [5]) to Banach space operators, with an alternate proof.

Proposition 2.1. For $A_{1} \in \mathrm{BL}\left(X_{1}\right), A_{2} \in \mathrm{BL}\left(X_{2}\right)$, consider the map $T: \mathrm{BL}\left(X_{1}, X_{2}\right) \rightarrow \mathrm{BL}\left(X_{1}, X_{2}\right)$ defined by

$$
T(B)=A_{2} B-B A_{1}, \quad B \in \mathrm{BL}\left(X_{1}, X_{2}\right) .
$$

Then $0 \notin \sigma(T)$ if and only if $\sigma\left(A_{1}\right) \cap \sigma\left(A_{2}\right)=\emptyset$.

For $A_{1} \in \mathrm{BL}\left(X_{1}\right)$ and $A_{2} \in \mathrm{BL}\left(X_{2}\right)$, define

$$
\operatorname{sep}\left(A_{1}, A_{2}\right)= \begin{cases}0 & \text { if } 0 \in \sigma(T), \\ 1 /\left\|T^{-1}\right\| & \text { otherwise, }\end{cases}
$$

where $T$ is as in Proposition 2.1. Using the standard perturbation theory arguments (cf. Kato [2]), we obtain the following result (see e.g. Nair [4], [5]).

Proposition 2.2. Let $A_{1}, V_{1} \in \mathrm{BL}\left(X_{1}\right)$ and $A_{2}, V_{2} \in \mathrm{BL}\left(X_{2}\right)$. Then

$$
\operatorname{sep}\left(A_{1}+V_{1}, A_{2}+V_{2}\right) \geq \operatorname{sep}\left(A_{1}, A_{2}\right)-\left\|V_{1}\right\|-\left\|V_{2}\right\| .
$$

In particular, if

$$
\operatorname{sep}\left(A_{1}, A_{2}\right)>0, \quad\left\|V_{1}\right\|+\left\|V_{2}\right\|<\operatorname{sep}\left(A_{1}, A_{2}\right),
$$


then the operator

$$
B \mapsto\left(A_{2}+V_{2}\right) B-B\left(A_{1}+V_{1}\right), \quad B \in \mathrm{BL}\left(X_{1}, X_{2}\right),
$$

is invertible.

For closed subsets $\Lambda_{1}$ and $\Lambda_{2}$ of the complex plane, define

$$
\operatorname{sep}\left(\Lambda_{1}, \Lambda_{2}\right):=\sup \left\{\operatorname{dist}\left(\lambda, \Lambda_{2}\right): \lambda \in \Lambda_{1}\right\} .
$$

We note that for $\omega>0$,

$$
\operatorname{sep}\left(\Lambda_{1}, \Lambda_{2}\right)<\omega \Leftrightarrow \Lambda_{1} \subseteq\left\{z: \operatorname{dist}\left(z, \Lambda_{2}\right)<\omega\right\} .
$$

Let $A \in \operatorname{BL}(X)$ and $\Lambda$ be a spectral set of $A$, i.e., $\Lambda$ is a subset of the spectrum $\sigma(A)$ of $A$ such that both $\Lambda$ and $\sigma(A) \backslash \Lambda$ are closed subsets of the complex plane. Then (cf. Taylor [10]) there exists an open subset $\Omega$ of the complex plane such that its boundary $\Gamma$ consists of a finite number of simple closed contours and

$$
\sigma(A) \cap(\Omega \cup \Gamma)=\Lambda .
$$

The range of the spectral projection (cf. Kato [2], Limaye [3])

$$
P_{\Lambda}=\frac{-1}{2 \pi i} \int_{\Gamma}(A-z I)^{-1} d z
$$

is called the spectral subspace of $A$ associated with the spectral set $\Lambda$.

Here is a characterization of a spectral subspace which is found to be useful in spectral approximation (cf. Nair [4], [5]).

Proposition 2.3. For $A \in \mathrm{BL}(X)$, let $P \in \mathrm{BL}(X)$ be a projection whose range $M=P(X)$ is invariant under $A$. Then $M$ is a spectral subspace of $A$ if and only if

$$
\sigma\left(\left.P A\right|_{P(X)}\right) \cap \sigma\left(\left.(I-P) A\right|_{(I-P)(X)}\right)=\emptyset,
$$

and in that case the associated spectral set is $\sigma\left(\left.P A\right|_{P(X)}\right)$ and

$$
\sigma(A)=\sigma\left(\left.P A\right|_{P(X)}\right) \cup \sigma\left(\left.(I-P) A\right|_{(I-P)(X)}\right) .
$$

3. Existence of the solution. Let $A_{1} \in \mathrm{BL}\left(X_{1}\right), A_{2} \in \mathrm{BL}\left(X_{2}\right), A_{3} \in$ $\mathrm{BL}\left(X_{1}, X_{2}\right)$ and $A_{4} \in \mathrm{BL}\left(X_{2}, X_{1}\right)$. Assume that

$$
\delta:=\operatorname{sep}\left(A_{1}, A_{2}\right)>0 .
$$

Let

$$
\gamma=\left\|A_{3}\right\|, \quad \eta=\left\|A_{4}\right\|, \quad \varepsilon=\eta \gamma / \delta^{2} .
$$

In the following we shall make use of the function

$$
g(t)= \begin{cases}1 & \text { if } t=0 \\ (1-\sqrt{1-4 t}) /(2 t) & \text { if } 0<t \leq 1 / 4\end{cases}
$$


It follows that $1 \leq g(t) \leq 2$ for $0 \leq t \leq 1 / 4$, and $s=g(t)$ satisfies the relation

$$
t s^{2}-s+1=0 .
$$

THEOREM 3.1. If $\varepsilon<1 / 4$, then the Riccati equation

$$
A_{2} R-R A_{1}=A_{3}+R A_{4} R
$$

has a unique solution $R \in \mathrm{BL}\left(X_{1}, X_{2}\right)$ which satisfies the relation

$$
\|R\| \leq \frac{\gamma}{\delta} g(\varepsilon) .
$$

Proof. By the assumption $\delta:=\operatorname{sep}\left(A_{1}, A_{2}\right)>0$, the operator $T$ on $\operatorname{BL}\left(X_{1}, X_{2}\right)$ defined by

$$
T(B)=A_{2} B-B A_{1}, \quad B \in \mathrm{BL}\left(X_{1}, X_{2}\right),
$$

is invertible. Let

$$
\mathcal{D}=\left\{B \in \mathrm{BL}\left(X_{1}, X_{2}\right):\|B\| \leq \frac{\gamma}{\delta} g(\varepsilon)\right\} .
$$

For $B \in \mathcal{D}$, let

$$
F(B)=T^{-1}\left(A_{3}+B A_{4} B\right) .
$$

Then we have

$$
\begin{aligned}
\|F(B)\| & \leq\left\|T^{-1}\right\|\left(\left\|A_{3}\right\|+\left\|A_{4}\right\| \cdot\|B\|^{2}\right) \leq \frac{1}{\delta}\left[\gamma+\eta\left(\frac{\gamma}{\delta} g(\varepsilon)\right)^{2}\right] \\
& =\frac{\gamma}{\delta}\left(1+\varepsilon(g(\varepsilon))^{2}\right)=\frac{\gamma}{\delta} g(\varepsilon) .
\end{aligned}
$$

Thus $F$ maps the complete metric space $\mathcal{D}$ into itself. Therefore, the proof will be completed once it is shown that $F: \mathcal{D} \rightarrow \mathcal{D}$ is a contraction. For this, let $B_{1}, B_{2} \in \mathcal{D}$ and observe that

$$
\begin{aligned}
F\left(B_{1}-B_{2}\right) & =T^{-1}\left(B_{1} A_{4} B_{1}-B_{2} A_{4} B_{2}\right) \\
& =T^{-1}\left[B_{1} A_{4}\left(B_{1}-B_{2}\right)+\left(B_{1}-B_{2}\right) A_{4} B_{2}\right] .
\end{aligned}
$$

Therefore,

$$
\begin{aligned}
\left\|F\left(B_{1}-B_{2}\right)\right\| & \leq\left\|T^{-1}\right\|\left(\left\|B_{1} A_{4}\right\|+\left\|A_{4} B_{2}\right\|\right)\left\|B_{1}-B_{2}\right\| \\
& \leq \frac{1}{\delta} \eta\left(\left\|B_{1}\right\|+\left\|B_{2}\right\|\right)\left\|B_{1}-B_{2}\right\| \leq 2 \varepsilon g(\varepsilon)\left\|B_{1}-B_{2}\right\| .
\end{aligned}
$$

Since $2 \varepsilon g(\varepsilon)=1-\sqrt{1-4 \varepsilon}<1$, it follows that $F$ is a contraction mapping on $\mathcal{D}$.

4. The iterative procedure. We keep the notation of the previous sections and the assumption $\delta>0$. Recall that then the operator $T$ is invertible. We also observe that if $\gamma=0$, then $R=0$ is the unique solution 
of the Riccati equation $A_{2} R-R A_{1}=A_{3}+R A_{4} R$. Therefore, hereafter, we assume that $\gamma>0$.

First we prove a technical result.

Proposition 4.1. Let $r_{1}=\gamma / \delta$ and $\varepsilon<1 / 4$. Then the relations

$$
\begin{gathered}
2 \eta r_{k}+2 \eta\left(r_{1}+\ldots+r_{k}\right)<\delta, \\
r_{k+1}:=\frac{\eta r_{k}^{2}}{\delta-2 \eta\left(r_{1}+\ldots+r_{k}\right)}<\frac{r_{k}}{2}
\end{gathered}
$$

hold iteratively.

Proof. Note that $4 \eta r_{1}=4 \varepsilon \delta<\delta$, so that $r_{2}$ is well defined and

$$
r_{2}=r_{1}\left(\frac{\eta r_{1}}{\delta-2 \eta r_{1}}\right)<\frac{r_{1}}{2} \text {. }
$$

Thus the result is proved for $k=1$. Assume the result for some $k=n-1$, $n \in\{2,3, \ldots\}$. Then for $k=n$ we have

$$
\begin{aligned}
2 \eta r_{k}+2 \eta\left(r_{1}+\ldots+r_{k}\right) & <2 \eta r_{1}\left(\frac{1}{2^{k-1}}+1+\frac{1}{2}+\frac{1}{2^{2}}+\ldots+\frac{1}{2^{k-1}}\right) \\
& =4 \eta r_{1}=4 \varepsilon \delta<\delta .
\end{aligned}
$$

Hence $r_{k+1}$ is well defined and

$$
r_{k+1}=\frac{\eta r_{k}^{2}}{\delta-2 \eta\left(r_{1}+\ldots+r_{k}\right)}=r_{k}\left(\frac{\eta r_{k}}{\delta-2 \eta\left(r_{1}+\ldots+r_{k}\right)}\right)<\frac{r_{k}}{2} .
$$

Theorem 4.2. Let $\varepsilon<1 / 4, A_{1}^{(0)}=A_{1}, A_{2}^{(0)}=A_{2}$ and $\left(r_{k}\right)$ be the sequence of nonnegative numbers defined in Proposition 4.1. Let $R_{1}$ be the unique element in $\mathrm{BL}\left(X_{1}, X_{2}\right)$ such that

$$
A_{2}^{(0)} R_{1}-R_{1} A_{1}^{(0)}=A_{3} .
$$

If $\varepsilon<1 / 4$, then the following hold iteratively.

(i) If $A_{1}^{(k)}=A_{1}^{(k-1)}-A_{4} R_{k}$ and $A_{2}^{(k)}=A_{2}^{(k-1)}-R_{k} A_{4}$, then

$$
\delta_{k}:=\operatorname{sep}\left(A_{1}^{(k)}, A_{2}^{(k)}\right)>\delta-2 \eta\left(r_{1}+\ldots+r_{k}\right)>0 .
$$

(ii) Let $R_{k+1}$ be the unique element in $\mathrm{BL}\left(X_{1}, X_{2}\right)$ such that

$$
A_{2}^{(k)} R_{k+1}-R_{k+1} A_{1}^{(k)}=R_{k} A_{4} R_{k} .
$$

Then

$$
\left\|R_{k+1}\right\| \leq \frac{\eta}{\delta-2 \eta\left(r_{1}+\ldots+r_{k}\right)}\left\|R_{k}\right\|^{2} \leq r_{k+1} .
$$

In particular,

$$
\left\|R_{k+1}\right\| \leq \frac{\eta}{\delta(1-4 \varepsilon)}\left\|R_{k}\right\|^{2}
$$


Proof. Clearly,

$$
\left\|R_{1}\right\|=\left\|T^{-1}\left(A_{3}\right)\right\| \leq \frac{\gamma}{\delta} .
$$

Then, by Propositions 2.2 and 4.1,

$$
\delta_{1}:=\operatorname{sep}\left(A_{1}^{(1)}, A_{2}^{(1)}\right) \geq \operatorname{sep}\left(A_{1}^{(0)}, A_{2}^{(0)}\right)-2 \eta r_{1}=\delta-2 \eta r_{1}>0 .
$$

Hence there exists a unique $R_{2} \in \operatorname{BL}\left(X_{1}, X_{2}\right)$ such that

$$
A_{2}^{(1)} R_{2}-R_{2} A_{1}^{(1)}=R_{1} A_{4} R_{1},
$$

and it satisfies

$$
\left\|R_{2}\right\| \leq \frac{\left\|A_{4}\right\| \cdot\left\|R_{1}\right\|^{2}}{\delta_{1}} \leq \frac{\eta}{\delta-2 \eta r_{1}}\left\|R_{1}\right\|^{2} \leq \frac{\eta r_{1}^{2}}{\delta-2 \eta r_{1}}=r_{2} .
$$

Assume the result for all integers up to $k-1, k \geq 2$. Then for $k$, define

$$
A_{1}^{(k)}=A_{1}^{(k-1)}-A_{4} R_{k}, \quad A_{2}^{(k)}=A_{2}^{(k-1)}-R_{k} A_{4} .
$$

Then $\left\|R_{k}\right\| \leq r_{k}$, and by Propositions 2.2 and 4.1,

$$
\delta_{k}:=\operatorname{sep}\left(A_{1}^{(k)}, A_{2}^{(k)}\right) \geq \delta_{k-1}-2 \eta r_{k} \geq \delta-2 \eta\left(r_{1}+\ldots+r_{k}\right)>0 .
$$

Hence there exists a unique $R_{k+1} \in \mathrm{BL}\left(X_{1}, X_{2}\right)$ such that

$$
A_{2}^{(k)} R_{k+1}-R_{k+1} A_{1}^{(k)}=R_{k} A_{4} R_{k} .
$$

Then

$$
\begin{aligned}
\left\|R_{k+1}\right\| & \leq \frac{\left\|A_{4}\right\| \cdot\left\|R_{k}\right\|^{2}}{\delta_{k}} \leq \frac{\eta}{\delta-2 \eta\left(r_{1}+\ldots+r_{k}\right)}\left\|R_{k}\right\|^{2} \\
& \leq \frac{\eta r_{k}^{2}}{\delta-2 \eta\left(r_{1}+\ldots+r_{k}\right)}=r_{k+1} .
\end{aligned}
$$

Since $r_{j+1}<r_{j} / 2$ for $j=0,1,2, \ldots$, we have

so that

$$
2 \eta\left(r_{1}+\ldots+r_{k}\right)=4 \eta r_{1}\left(1-\frac{1}{2^{k}}\right) \leq 4 \varepsilon \delta
$$

$$
\left\|R_{k+1}\right\| \leq \frac{\eta}{\delta(1-4 \varepsilon)}\left\|R_{k}\right\|^{2}
$$

Next we obtain an estimate for the error $\left\|R-\left(R_{1}+\ldots+R_{k}\right)\right\|$, and also specify an additional condition under which $\left\|R-\left(R_{1}+\ldots+R_{k}\right)\right\| \rightarrow 0$ as $k \rightarrow \infty$. For this purpose let $R^{(0)}=R$ and for $k \in\{1,2, \ldots\}$, let $R^{(k)}=$ $R^{(k-1)}-R_{k}$, i.e.,

$$
R^{(k)}=R-\left(R_{1}+\ldots+R_{k}\right) .
$$

Theorem 4.3. Let $\varepsilon<1 / 4$. Then, for $k=1,2, \ldots$,

Moreover,

$$
\left\|R^{(k)}\right\| \leq \frac{\eta}{\delta(1-4 \varepsilon)}\left\|R^{(k-1)}\right\|^{2} .
$$

$$
\left\|R^{(k)}\right\| \leq \frac{\gamma}{\delta} g(\varepsilon) \beta^{2^{k}-1}, \quad \beta=\frac{\varepsilon}{1-4 \varepsilon} g(\varepsilon) .
$$


If , in addition, $\varepsilon<3 / 16$, then

$$
\left\|R^{(k)}\right\| \rightarrow 0 \quad \text { as } k \rightarrow \infty .
$$

Proof. First we note that the Riccati equation

$$
A_{2} R-R A_{1}=A_{3}+R A_{4} R
$$

takes the form

$$
A_{2}^{(k)} R^{(k)}-R^{(k)} A_{1}^{(k)}=R_{k} A_{4} R_{k}+R^{(k)} A_{4} R^{(k)}
$$

for $k=1,2, \ldots$, where $A_{1}^{(k)}$ and $A_{2}^{(k)}$ are defined iteratively as in Theorem 4.2. Thus, considering the the map

$$
T_{k}: B \mapsto A_{2}^{(k)} B-B A_{1}^{(k)}, \quad B \in \operatorname{BL}\left(X_{1}, X_{2}\right),
$$

for $k=1,2, \ldots$, it follows that

$$
R^{(k)}=T_{k}^{-1}\left(R_{k} A_{4} R_{k}+R^{(k)} A_{4} R^{(k)}\right)=R_{k+1}+T_{k}^{-1}\left(R^{(k)} A_{4} R^{(k)}\right),
$$

so that

$$
\begin{aligned}
R^{(k)} & =R^{(k-1)}-R_{k}=\left[R_{k}+T_{k-1}^{-1}\left(R^{(k-1)} A_{4} R^{(k-1)}\right)\right]-R_{k} \\
& =T_{k-1}^{-1}\left(R^{(k-1)} A_{4} R^{(k-1)}\right) .
\end{aligned}
$$

Hence, from the estimate for $\operatorname{sep}\left(A_{1}^{(k)}, A_{2}^{(k)}\right)$ from Theorem 4.2,

$$
\left\|R^{(k)}\right\| \leq\left\|T_{k-1}^{-1}\right\| \cdot\left\|A_{4}\right\| \cdot\left\|R^{(k-1)}\right\|^{2} \leq \frac{\eta}{\delta(1-4 \varepsilon)}\left\|R^{(k-1)}\right\|^{2} .
$$

Now let

$$
\alpha=\frac{\eta}{\delta(1-4 \varepsilon)}, \quad r=\frac{\gamma}{\delta} g(\varepsilon) .
$$

Recall from Theorem 3.1 that

$$
\left\|R^{(0)}\right\|=\|R\| \leq \frac{\gamma}{\delta} g(\varepsilon)=r .
$$

Then from the relation $\left\|R^{(k)}\right\| \leq \alpha\left\|R^{(k-1)}\right\|^{2}$ it follows that

$$
\left\|R^{(k)}\right\| \leq r \beta^{2^{k}-1}, \quad \beta:=\alpha r=\frac{\varepsilon}{1-4 \varepsilon} g(\varepsilon),
$$

for $k=0,1,2, \ldots$ Note that $\beta<1$ if and only if $\varepsilon<3 / 16$.

Remark. Denote the sequences of approximations of $R$ obtained by our procedure (1.4) and the Newton's iteration $(1.3)$ by $\left(S_{k}\right)$ and $\left(\widetilde{S}_{k}\right)$ respectively. Then the error bound obtained in Theorem 4.3 based on (1.4) can be written using the order function as

$$
\left\|R-S_{k}\right\|=O\left(\beta^{2^{k}-1}\right), \quad \beta=\frac{\varepsilon}{1-4 \varepsilon} g(\varepsilon),
$$

whereas the result obtained by Demmel [1] for $\varepsilon<1 / 12$ based on (1.3) is (cf. Nair [6], Theorem 4.2) 


$$
\left\|R-\widetilde{S}_{k}\right\|=O\left(\mu^{2^{k}-1}\right), \quad \mu=\frac{3}{2} \varepsilon g(\varepsilon) .
$$

We may observe that $\beta<\mu$ whenever $\varepsilon<1 / 12$. Thus, our result not only requires weaker assumptions on the coefficient operators, but also the estimate obtained is better for those values of $\varepsilon$ for which Demmel's result is applicable.

5. Application to spectral variation. Let $A: X \rightarrow X$ be a bounded linear operator on a complex Banach space $X$ and $M_{0}$ be a closed subspace of $X$. We would like to impose conditions on $M_{0}$ such that it is close to a spectral subspace, and then obtain a sequence $\left(M_{k}\right)$ of closed subspaces of $X$ which converges to a spectral subspace in the sense of gap.

For $M_{0}$ to be close to a spectral subspace of $A$ we assume the following.

Assumption 1. There exists a closed subspace $N_{0}$ such that

$$
X=M_{0} \oplus N_{0} .
$$

Let $P_{0}: X \rightarrow X$ be the projection onto $M_{0}$ along $N_{0}$ and let

$$
\left[\begin{array}{ll}
A_{11} & A_{12} \\
A_{21} & A_{22}
\end{array}\right]
$$

be the matrix representation of $A$ with respect to the decomposition (5.1), i.e., if we take

$$
Q_{1}=P_{0}, \quad Q_{2}=I-P_{0}, \quad X_{1}=Q_{1}(X), \quad X_{2}=Q_{2}(X),
$$

the operators $A_{i j}: X_{j} \rightarrow X_{i}$ are defined by

$$
A_{i j} x=Q_{i} A x, \quad x \in X_{j} .
$$

Recall that

- $M_{0}$ is invariant under $A$ if and only if $A_{21}=0$, and

- $M_{0}$ is a spectral subspace of $A$ if and only if $\sigma\left(A_{11}\right) \cap \sigma\left(A_{22}\right)=\emptyset$ and $A_{21}=0$.

Assumption 2.

$$
\sigma\left(A_{11}\right) \cap \sigma\left(A_{22}\right)=\emptyset .
$$

This assumption is equivalent to the condition

$$
\delta:=\operatorname{sep}\left(A_{11}, A_{22}\right)>0 .
$$

It is to be mentioned that the condition (5.2) is satisfied if, for example, $A$ is an "approximation" of another operator $A_{0}$, and $M_{0}$ is a spectral subspace of $A_{0}$ with corresponding spectral projection $P_{0}$. To see this suppose that

$$
A=A_{0}+V
$$


and let

$$
\left[\begin{array}{ll}
A_{11}^{(0)} & A_{12}^{(0)} \\
A_{21}^{(0)} & A_{22}^{(0)}
\end{array}\right], \quad\left[\begin{array}{ll}
V_{11} & V_{12} \\
V_{21} & V_{22}
\end{array}\right]
$$

be the matrix representations (w.r.t. the decomposition (5.1)) of $A_{0}$ and $V$ respectively. Since $P_{0}$ is a spectral projection of $A_{0}$, we have

$$
A_{12}^{(0)}=0=A_{21}^{(0)} \quad \text { and } \quad \delta_{0}:=\operatorname{sep}\left(A_{11}^{(0)}, A_{22}^{(0)}\right)>0 .
$$

Thus

$$
\left[\begin{array}{ll}
A_{11} & A_{12} \\
A_{21} & A_{22}
\end{array}\right]=\left[\begin{array}{cc}
A_{11}^{(0)}+V_{11} & V_{12} \\
V_{21} & A_{22}^{(0)}+V_{22}
\end{array}\right] .
$$

By Proposition 2.2, it follows that $\delta \geq \delta_{0}-\left\|V_{11}\right\|-\left\|V_{22}\right\|$.

Hence Assumption 2 is satisfied if

$$
\left\|V_{11}\right\|+\left\|V_{22}\right\|<\delta_{0} .
$$

Note that if the last inequality is satisfied, then

$$
\varepsilon=\frac{\left\|A_{21}\right\| \cdot\left\|A_{12}\right\|}{\delta^{2}} \leq \frac{\left\|V_{21}\right\| \cdot\left\|V_{12}\right\|}{\left(\delta_{0}-\left\|V_{11}\right\|-\left\|V_{22}\right\|\right)^{2}} .
$$

If we use the notation

$$
A_{1}=A_{11}, \quad A_{2}=A_{22}, \quad A_{3}=-A_{21}, \quad A_{4}=A_{12},
$$

then the definitions of $\delta$ and $\varepsilon$ in this section agree with those in the previous section.

Suppose $\varepsilon<1 / 4$, and $R$ and $R_{k}$ are as in the last section. Define

$$
P=P_{0}+R P_{0}, \quad M=P(X),
$$

and for $k=1,2, \ldots$, let

$$
P_{k}=P_{0}+\left(R_{1}+\ldots+R_{k}\right) P_{0}, \quad M_{k}=P_{k}(X) .
$$

Then, by Theorem 4.3, taking $M_{k}=P_{k}(X)$, we have

$$
\operatorname{sep}\left(M_{k}, M\right) \leq \frac{\gamma}{\delta} g(\varepsilon) \beta^{2^{k}-1}, \quad \operatorname{gap}\left(M_{k}, M\right) \leq\left\|P_{0}\right\| \frac{\gamma}{\delta} g(\varepsilon) \beta^{2^{k}-1},
$$

for $k=1,2, \ldots$ If, in addition, $\varepsilon<3 / 16$, then

$$
\operatorname{sep}\left(M_{k}, M\right) \rightarrow 0, \quad \operatorname{gap}\left(M_{k}, M\right) \rightarrow 0 \quad \text { as } k \rightarrow \infty .
$$

It can be seen that

$$
\begin{aligned}
\left.P A\right|_{P(X)} & =\left.P\left(A_{11}+A_{12} R\right)\right|_{P(X)}, \\
\left.(I-P) A\right|_{(I-P)(X)} & =\left.(I-P)\left(A_{22}+R A_{12}\right)\right|_{(I-P)(X)},
\end{aligned}
$$

so that

$$
\sigma\left(\left.P A\right|_{P(X)}\right)=\sigma\left(A_{11}+A_{12} R\right), \quad \sigma\left(\left.(I-P) A\right|_{(I-P)(X)}\right)=\sigma\left(A_{22}+R A_{12}\right) .
$$

Hence, by Proposition 2.3, $M=P(X)$ is a spectral subspace of $A$ with the associated spectral set $\Lambda:=\sigma\left(A_{11}+A_{12} R\right)$ provided 


$$
\sigma\left(A_{11}+A_{12} R\right) \cap \sigma\left(A_{22}+R A_{12}\right)=\emptyset .
$$

But this is true because

$$
\operatorname{sep}\left(A_{11}+A_{12} R, A_{22}+R A_{12}\right) \geq \delta-2 \eta\|R\| \geq \delta(1-2 \varepsilon g(\varepsilon))>0 .
$$

Next let

$$
B=A_{11}+A_{12} R, \quad B_{k}=A_{11}+A_{12} S_{k},
$$

where $S_{k}=R_{1}+\ldots+R_{k}, k=1,2, \ldots$ Then we have

$$
\left\|B-B_{k}\right\| \leq\left\|R-\left(R_{1}+\ldots+R_{k}\right)\right\| \rightarrow 0 \quad \text { as } k \rightarrow \infty .
$$

Thus, by the upper semicontinuity of the spectrum (cf. Kato [2], Chapter IV, Remark 3.3), it follows that

$$
\operatorname{sep}\left(\sigma\left(B_{k}\right), \sigma(B)\right) \rightarrow 0 \quad \text { as } k \rightarrow \infty .
$$

Acknowledgements. I thank the referees for many useful comments on the earlier versions of this paper.

\section{References}

[1] J. W. Demmel, Three methods for refining invariant subspaces, Computing 38 (1987), 43-57.

[2] T. Kato, Perturbation Theory for Linear Operators, 2nd ed., Springer, 1976.

[3] B. V. Limaye, Spectral Perturbation and Approximation with Numerical Experiments, Proc. Centre for Mathematical Analysis, Australian National Univ., Vol. 13, 1987.

[4] M. T. Nair, Approximation and localization of eigenelements, Ph.D. thesis, I.I.T. Bombay, 1984.

[5] -, Approximation of spectral sets and spectral subspaces in Banach spaces, J. Indian Math. Soc. 54 (1989), 1-14.

[6] -, On iterative refinements for spectral sets and spectral subspaces, Numer. Funct. Anal. Optim. 10 (1989), 1019-1037.

[7] - Computable error estimates for Newton's iterations for refining invariant subspaces, Indian J. Pure Appl. Math. 21 (1990), 1049-1054.

[8] M. Rosenblum, On the operator equation $B X-X A=Q$, Duke Math. J. 23 (1956), 263-270.

[9] G. W. Stewart, Error bounds for approximate invariant subspaces of closed operators, SIAM J. Numer. Anal. 8 (1971), 796-808.

[10] A. E. Taylor, Spectral theory of closed distributive operators, Acta Math. 84 (1950), $189-224$.

Department of Mathematics

Indian Institute of Technology Madras

Chennai 600 036, India

E-mail: mtnair@iitm.ac.in 